\title{
Thermostabilization of Hyaluronidase by Chondroitin Ligands in Molecular Docking
}

\author{
Alexander Vasilievich Maksimenko*, Robert Shavlovich Beabealashvili \\ Institute of Experimental Cardiology, National Research Medical Center for Cardiology, Moscow, Russia
}

Email address:

alex.v.maks@mail.ru (A.V. Maksimenko)

${ }^{*}$ Corresponding author

\section{To cite this article:}

Alexander Vasilievich Maksimenko, Robert Shavlovich Beabealashvili. Thermostabilization of Hyaluronidase by Chondroitin Ligands in Molecular Docking. Cardiology and Cardiovascular Research. Vol. 3, No. 2, 2019, pp. 37-44. doi: 10.11648/j.ccr.20190302.14

Received: May 28, 2019; Accepted: July 15, 2019; Published: July 30, 2019

\begin{abstract}
Speedup of present high-molecular drug derivatives developments is based on the computational methods harmonic application. Such glycosidase as hyaluronidase has been functionated among multifarious glycosaminoglycan microenvironment in blood circulation of organism. It is important for elucidation of action mechanism of biosystem components (on vascular wall) and productive obtaining of hyaluronidase derivatives of cardiological destination the using of computer aided calculations for investigation of protein-glycosaminoglycan interactions. The aim of our study became the molecular docking fulfillment for 3D model of bovine testicular hyaluronidase with short-chain dimer and trimer chondroitin ligands. We used the molecular docking of hyaluronidase with chondroitin ligands for theoretical determination of biocatalyst conformational stability. At temperatures higher than $300 \mathrm{~K}$ free/native hyaluronidase (without ligands) displayed conformational transitions leading to its inactivation. Predominant manifestation of these transitions was an irreversible attraction developing between the protein area proximate to Glu-105, on the one hand, and to Arg-59 and Arg-96, on the other. Lowering of temperature did not restore the initial 3D structure of hyaluronidase. Binding of chondroitin ligands at ch 6 , ch 3 and ch 1 sites stabilized the enzyme, increasing its denaturation temperature by $10^{\circ} \mathrm{C}$. The highest degree of stabilization was achieved after chondroitin binding to ch 6 . This effect was higher than that for chondroitin sulfate trimers concerning the enzyme inhibition by heparin tetramer, which requires binding of 4-5 chondroitin sulfate ligands to the enzyme surface. Molecular docking of 3D model of bovine testicular hyaluronidase with chondroitin dimers and trimers has revealed eight sites for their binding to the enzyme surface. At biological concentrations of these ligands the most important binding sites for them are ch 6 , ch 3 and ch 1 . Binding at these sites induces a reversible deformation of the protein 3D structure. Interactions between 3D model of bovine hyaluronidase with chondroitin ligands are based predominantly on electrostatic forces. Chondroitin ligands stabilized 3D structure of hyaluronidase after binding predominantly at chondroitin 6 as well as chondroitin 3 and chondroitin 1 positions and their effect was higher than chondroitin sulfate upon enzyme inhibition by heparin tetramer. Stabilizing effects of chondroitin and chondroitin sulfate ligands are fundamental for further direct theoretical comparative investigation of impact produced by these ligands.
\end{abstract}

Keywords: Bovine Testicular Hyaluronidase, Protein Space Structure, Glycosaminoglycan Ligands, Chondroitin, Molecular Docking, Enzyme Structure Stabilization

\section{Introduction}

The use of high-molecular-weight compounds opened a new page in the development of drug therapeutic strategies against a wide variety of human diseases. Enzyme derivatives occupy an important place among these compounds. Plasminogen activators, antioxidant biocatalysts and antibodies have been extensively used in cardiology for thrombolysis, oxidative stress blocking and targeted drug delivery $[1,2]$. Broad application of enzymes in medical practice boosted the research into the mechanisms responsible for their effects in biological systems and triggered the search for modification of enzymes to increase their effectiveness and improve protocols for their clinical application [3]. The need for new generation medicines instigated extensive theoretical and experimental studies. Harmony between these studies verified by consistence of their results markedly improves their productivity. Theoretical studies are 
increasingly based on calculational methods which can speed up and reduce the cost of biomedical investigations. It seems to be reasonable to employ computational methods for study of protein-glycosaminoglycan binding for its modeling which often becomes a single tool to provide a better and deeper insight into glycosaminoglycan interactions $[4,5]$. Molecular docking of enzymes with various ligands occurring in their native microenvironment offers a promising way to achieve these goals. The molecular dynamics method was shown provide modeling of all degrees of freedom in a protein-ligand complex and draws special attention to enzyme structure flexibility $[6,7]$. Computer aided calculations promoted the study of glycosaminoglycans of the glycocalyx, an important constituent of the vascular wall protective layer. Vascular endothelium and its glycocalyx have been considered as double protective stratum of vessel lumen at present $[8,9]$. These developments prompted us to use consecutive molecular docking for modeling (computer simulation) of interactions between hyaluronidase, an enzyme involved in regulations of the glycocalyx, and glycosaminoglycans of its native microenvironment.

Microenvironment can be regarded as a regulatory factor of hyaluronidase activity, which is important for understanding the function of enzymes with trans- and endoglycosidase activities as well as directed therapeutic modulation of tissue permeability. Hyaluronidase microenvironment can be modeled by covalent binding of glycans. A high-degree modification with dextran (96-98\%) renders hyaluronidase resistant to heparin-induced inhibition [10]. A low-degree $(\sim 15 \%)$ modification with dextran markedly reduces endoglycosidase activity of hyaluronidase inhibited by the excess of heparin. It can be suggested that sulfatation of the neutral homopolymeric dextran $[\operatorname{Glc}(\alpha 1-6)] \mathrm{n}$ increases the rigidity of its structure by inducing electrostatic interactions and enhances its inactivating effect on hyaluronidase [11]. Testicular hyaluronidase hydrolyzes hyaluronan with greater efficiency than chondroitin sulfate [12], thus empathizing the effect of sulfatation on the interacting agents. Moreover, the activity of hyaluronidase is similar towards hyaluronan and chondroitin $(\mathrm{CH})$ which lack sulfogroups [13]. Hyaluronidase is inactivated by covalent binding of the copolymeric glycosaminoglycans (GAG) heparin and dermatan sulfate, while binding with chondroitin sulfate (CHS), a polymeric GAG, increases the enzyme resistance to heparin-induced inhibition [11]. Contralateral effects of dextran and dextran sulfate on hyaluronidase suggest the presence of regions/centers which limit interactions with these polysaccharides $[10,11]$.

Investigation of thin/delicate interactions occurring in surface contacts between hyaluronidase and GAG is hampered by high-molecular-weight nature of GAG. Molecular docking of hyaluronidase with short chain GAG fragments/ligands seems to be a reasonable and substantiated approach to this problem. Docking of bovine testicular hyaluronidase $(\mathrm{BTH})$ with CHS trimer and HP tetramer has revealed eight binding sites for GAG ligands on the enzyme surface [14]. HP inactivates BTH by entering the enzyme active site, while CHS produces a protective effect by occupying 4-5 binding sites, which abolishes BTH inactivation by HP. This finding demonstrates that BTH stability is regulated by competitive binding of various ligands based predominantly on electrostatic forces. Consecutive addition of detail to docking, evaluation of the effect produced by sulfatation of BTH and discovery of interaction prospective for design of novel protein drugs motivated us to perform molecular docking between BTH and short-chain $\mathrm{CH}$ ligands (dimer, trimer).

The goal of our investigation was the perform of molecular docking for 3D model of bovine testicular hyaluronidase with dimer and trimer chondroitin ligands for elucidation of functioning regulation this enzyme among short-chain glycosaminoglycan fragments of biocatalyst microenvironment.

\section{Calculations}

Earlier we constructed the 3D model of native (free from ligands) BTH [15] and used this model for molecular docking with $\mathrm{CH}$ ligands. 3D-model of free BTH was docked with $\mathrm{CH}$ dimer and trimer using UCSF Chimera and DOCK software [16-18]. UCSF Chimera was used to produce images and visualization, DOCK was applied to perform docking of low-molecular mass molecules into a state macromolecule. $\mathrm{CH}$ ligands were chosen from Pubchem. ncbi. nlm. nih. gov using dimer and trimer ([-- 4) $\operatorname{GlcA}(\beta 1-3) \operatorname{GalNAc}(\beta 1--] \mathrm{n})$, where $n$ is equal to 2 or 3 , respectively [19]). $\mathrm{CH}$ docking on BTH molecule surface (a mixture of 10 dimers and 10 trimers per BHT molecule) was run 100 times at $\mathrm{pH} 7.5,0.1 \mathrm{M} \mathrm{NaCl}$, $18 \mathrm{M} \mathrm{HOH}$, at 310,320 and $340 \mathrm{~K}$. Heat inactivation of free BTH (without $\mathrm{CH}$ ligands) was calculated under the same conditions. Reverse dynamics of 3D structure of the enzyme was examined after lowering temperature from the given values to $295 \mathrm{~K}$.

\section{Results and Discussion}

Molecular docking of 3D-model of BTH [15] with $\mathrm{C} \mathrm{H}$ dimers and trimers (nonsulfatated GAG ([-- 4) GlcA( $\beta 1-)$ GalNAc ( $\beta 1--] \mathrm{n}$, where $\mathrm{n}$ is 2 or 3 , respectively [19]) has revealed 8 binding sites/positions for these ligands on the enzyme surface (Figure 1). These sites are quite similar but not identical to the binding sites for CHS trimer and HP tetramer [14]. BTH surface was saturated with $\mathrm{CH}$ at a maximum binding of 4 ligands per molecule. Three ligands were bound in $25 \%$ runs, binding of 2 ligands was very rare, and that of 5 ligands was never observed. Positions ch 1, ch 3 and ch 6 were almost always occupied (numeration is the same as in docking with CHS ligands [14]). Free binding energy was the highest $(-/ 620-690 / \mathrm{kcal} / \mathrm{mol})$ at ch 6 site. At ch 3 site it was $-/ 310-420 / \mathrm{kcal} / \mathrm{mol}$ and $-/ 280-320 / \mathrm{kcal} / \mathrm{mol}$ at $\mathrm{ch} 1$. Sites ch $2(150-180 \mathrm{kcal} / \mathrm{mol})$ and $\mathrm{ch} 7$ (120-140 kcal mol) with equal probability could be the binding sites for a fourth $\mathrm{CH}$ ligand. Centers ch 8 and ch 5 were occupied quite rarely, ch 4 was occupied only once. It should be noted that the 
energy of free $\mathrm{CH}$ ligands in $\mathrm{NaCl}$ aqueous solution (2 and 3 sodium cations in the proximity of $\mathrm{CH}$ dimer and trimer, respectively) for the trimer is roughly $-320 \mathrm{kcal} / \mathrm{mol}$ and $(180-320) \mathrm{kcal} / \mathrm{mol}$ for the dimer. Comparison of these values shows that ch 6 , ch 3 and ch 1 sites (Figure 2) are most interesting from the research viewpoint, concerning biological concentrations of $\mathrm{CH}$. Other binding sites can be ignored. 3D-structure records for BTH after binding $\mathrm{CH}$ ligands at these positions (for tens ps at $320 \mathrm{~K}$ ) demonstrate conformational changes that distort the enzyme structure, specifically, by narrowing the entrance into the active site between Glu-105 and Arg-59, Arg-96 amino acid residues
(Figures 3 and 4). These changes, however, were reversible, i. e., lowering of temperature restored the initial structure of the enzyme (Figure 5). It is noteworthy that $\mathrm{CH}$ binding at $\mathrm{ch} 6$, ch 3 and ch 1 did not induce any noticeable and irreversible changes in the enzyme conformation (Figures 2-5). Previously we demonstrated a stabilizing effect of substrate (dodecasaccharide hyaluronan) on BTH structure [14]. Bearing in mind that only ch 1 , ch 3 and ch 6 sites can be occupied by $\mathrm{CH}$ ligands with high probability, it seems reasonable to evaluate the effects of these ligands on the enzyme structure.

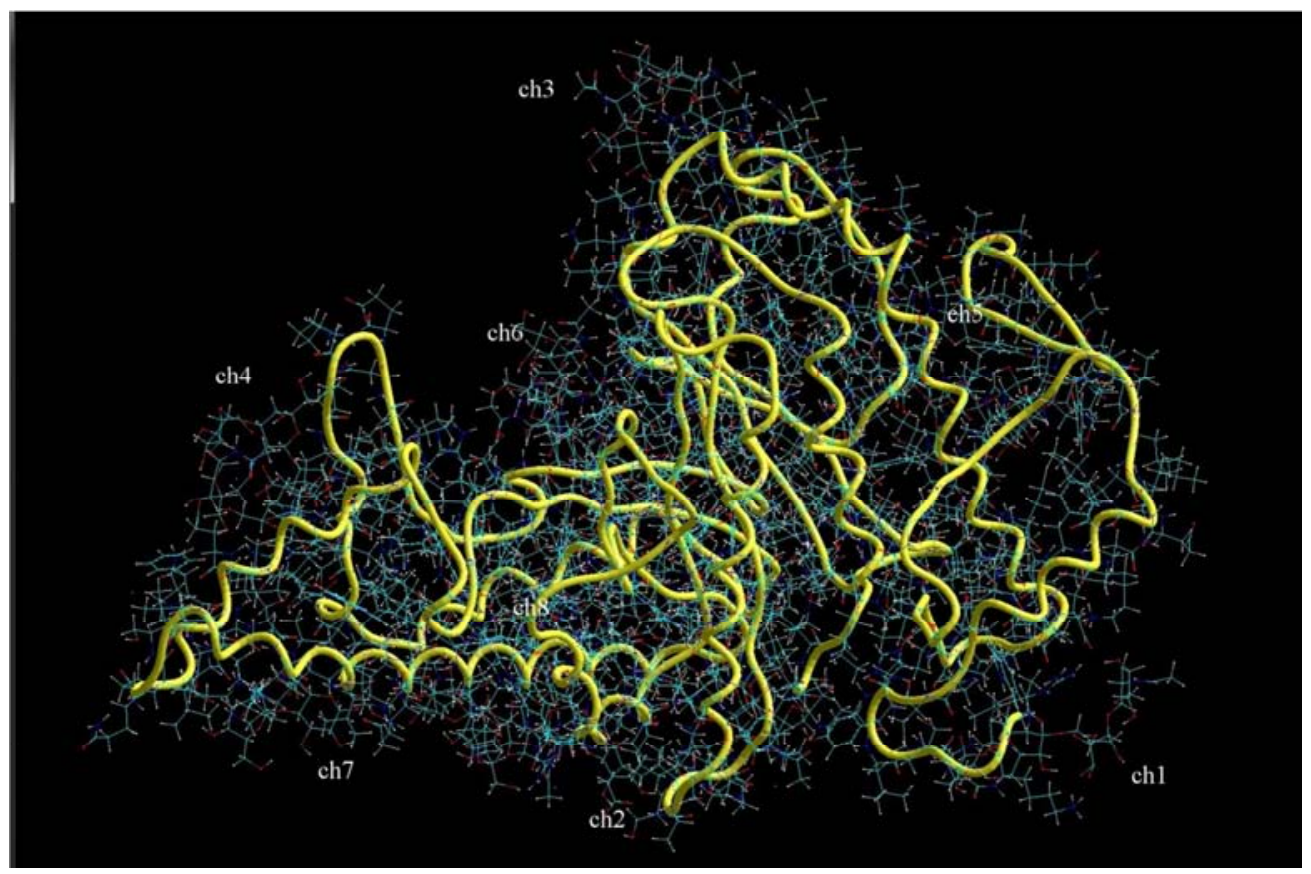

Figure 1. Binding of chondroitin (CH) dimers and trimers at 8 sites/positions (ch 1, ch 2, ch 3, etc.) on the surface of bovine testicular hyaluronidase (BTH).

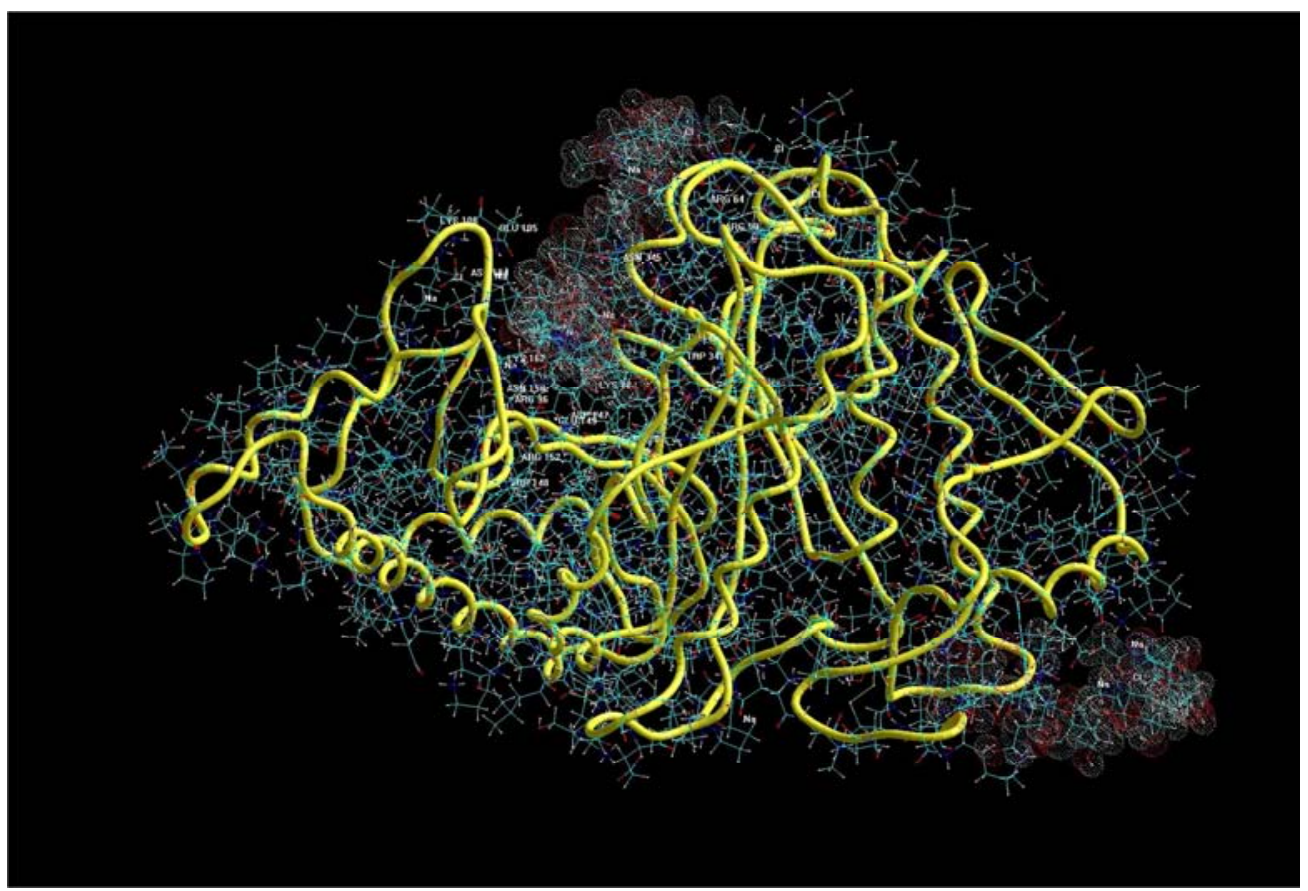

Figure 2. Binding of CH ligands at ch 1, ch 3 and ch 6 sites/positions (denoted with mesh) $310 \mathrm{~K}, 10 \mathrm{ps}$. 


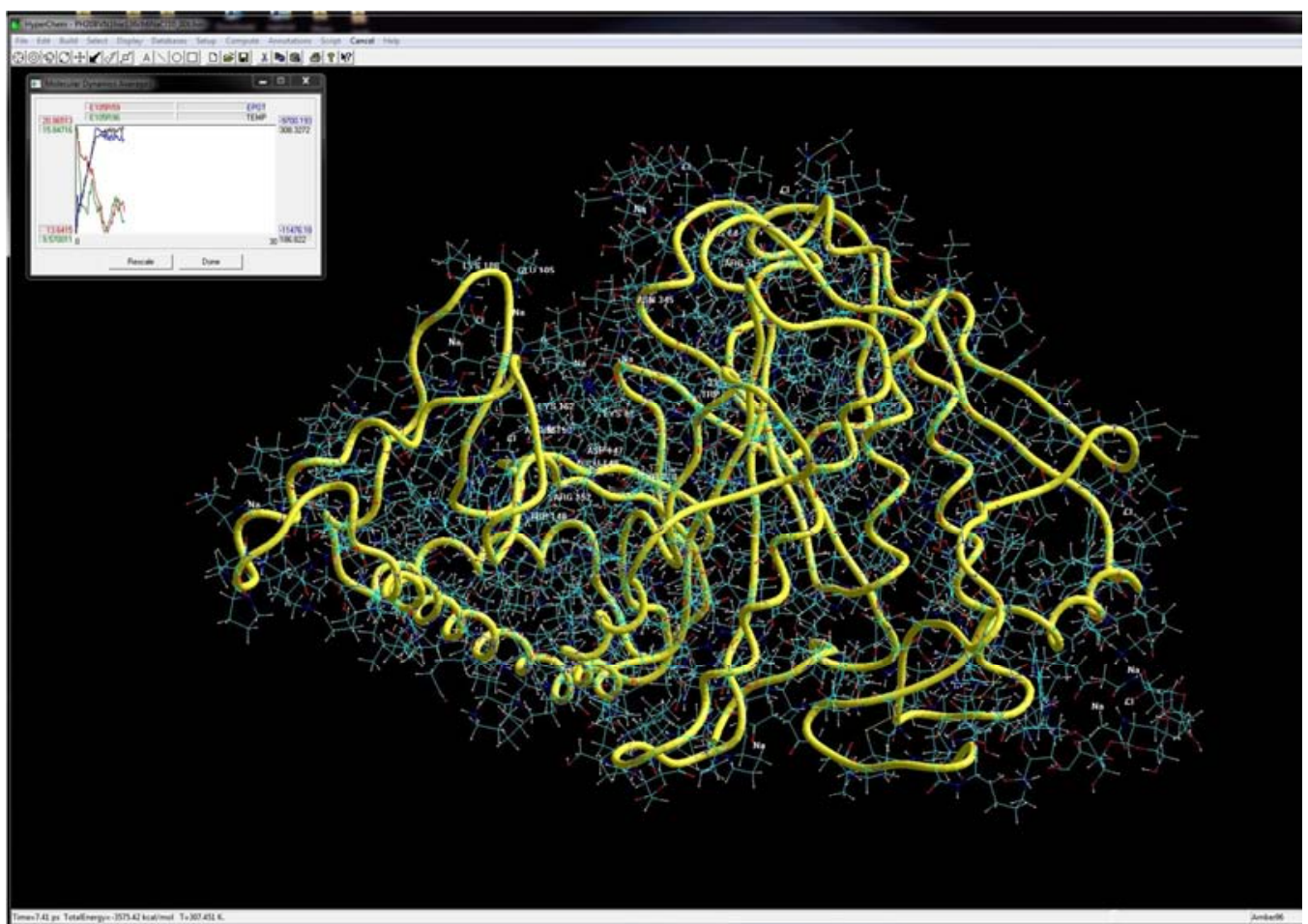

Figure 3. BTH structure with 3 CH ligands bound at ch 1, ch 3 and ch $6(310 K, 7 \mathrm{ps})$.

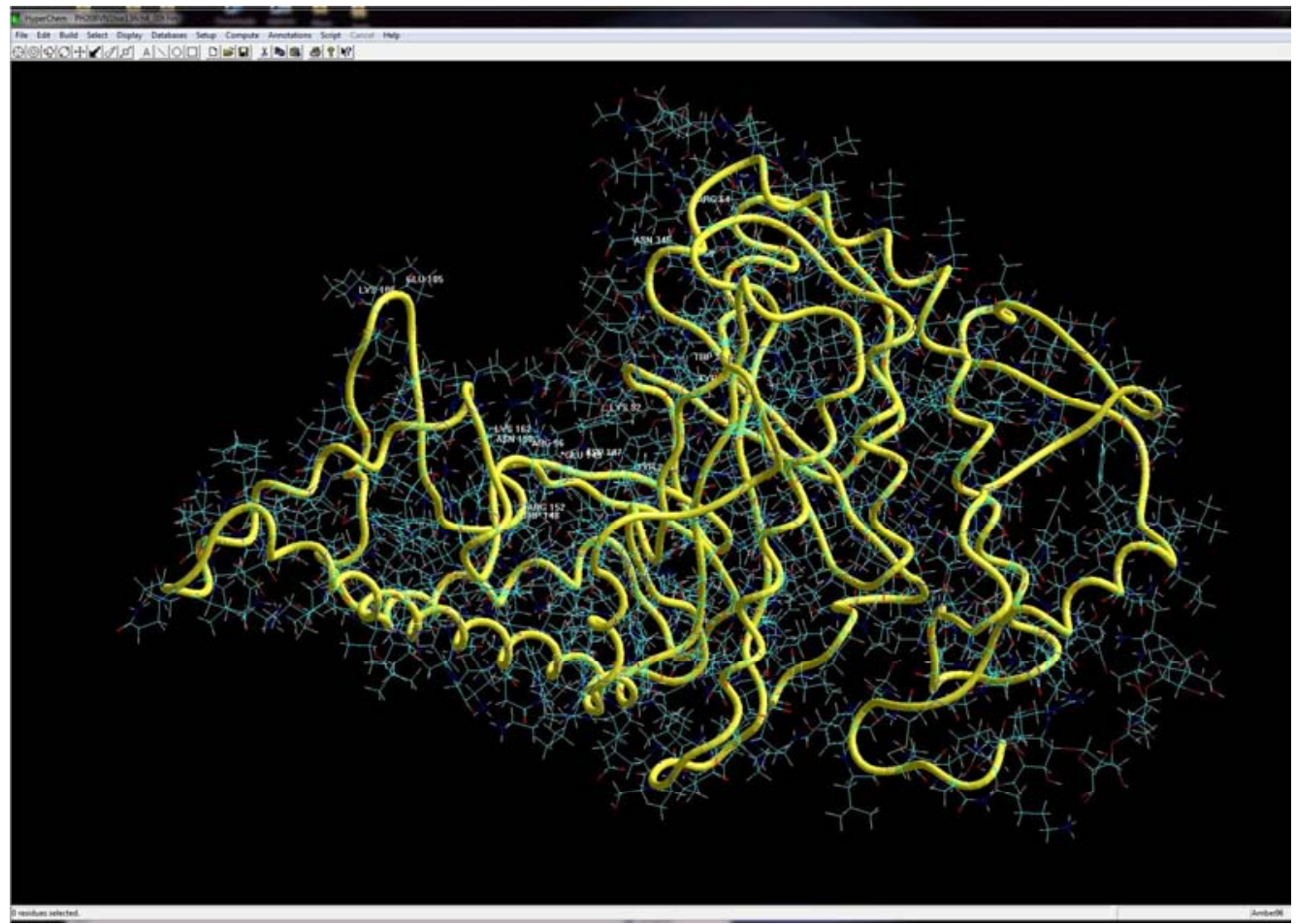

Figure 4. BTH structure at the initial calculational time ( $(0.17 \mathrm{ps})$ with CH ligands bound at ch 1 , ch 3 and ch 6 at $310 \mathrm{~K}$. Compare with the enzyme structures in Figures 2 and 3).

Structural deformation of free BHT (without $\mathrm{CH}$ ligands) was observed at $37^{\circ} \mathrm{C} / 300 \mathrm{~K}$. With time ( $35 \mathrm{ps)}$ an attraction of negatively charged area at Glu-105 to positively charged areas at Arg-59 and Arg-96 has developed (as in the presence of the ligands at ch 1, ch 3 and ch 6 sites in Figures 2 and 3), which led to narrowing and blockade of entrance to the enzyme active site at $340 \mathrm{~K}$ (Figures 6, 7). These changes in
BTH conformation occurring in the absence of ligands were irreversible, i. e., the initial molecular structure was not restored after temperature was lowered (Figure 8). The enzyme was inactivated by heat. Special attention should be paid to changes in the distance between amino acids at $320 \mathrm{~K}$ with time (ns, Figure 9). 


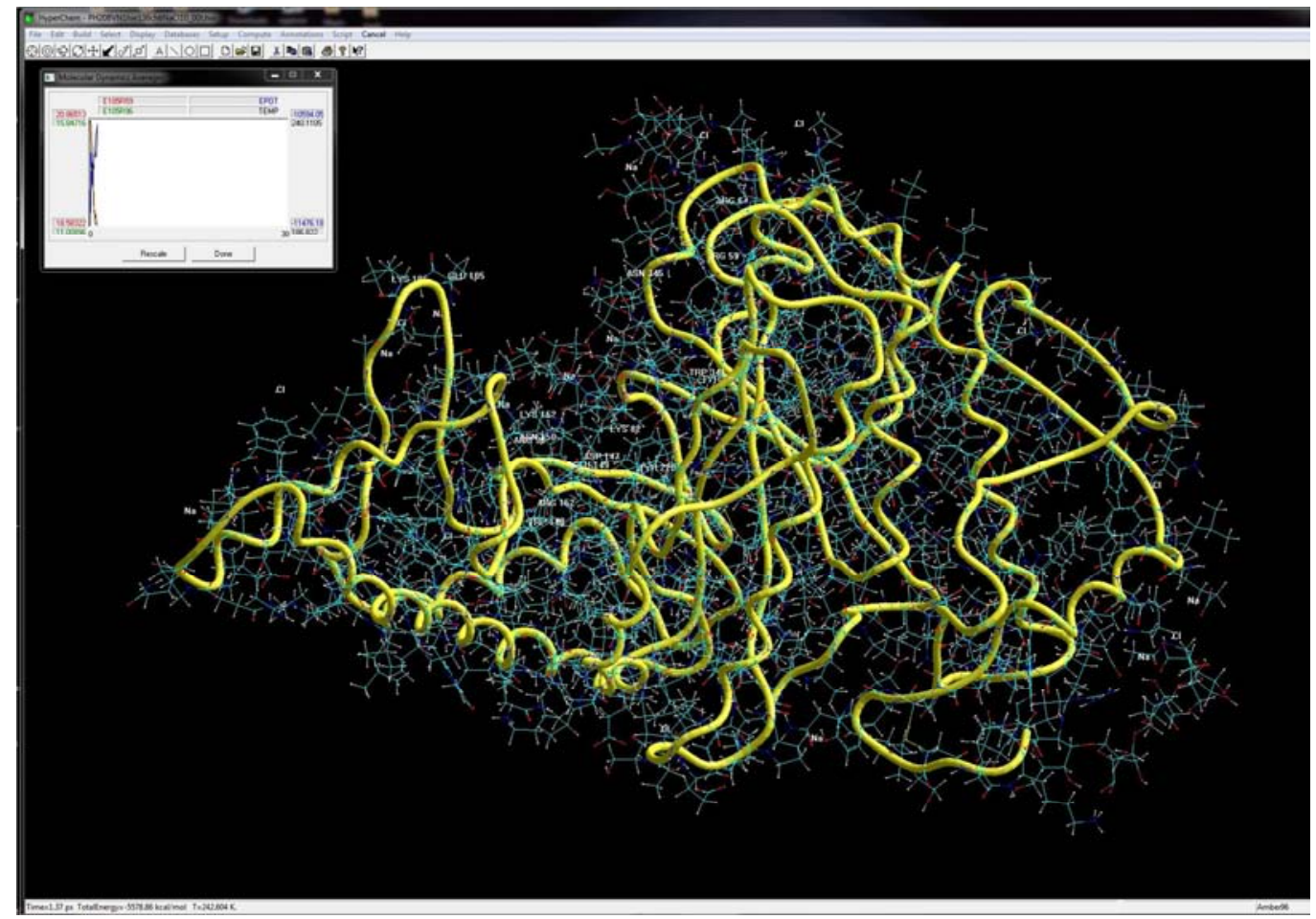

Figure 5. BTH structure with CH ligands bound at ch 1, ch 3 and ch 6 after lowering temperature to $295 \mathrm{~K}, 1.4$ ps. Restoration of the initial structure. Compare with structures in Figures 4 and 5).

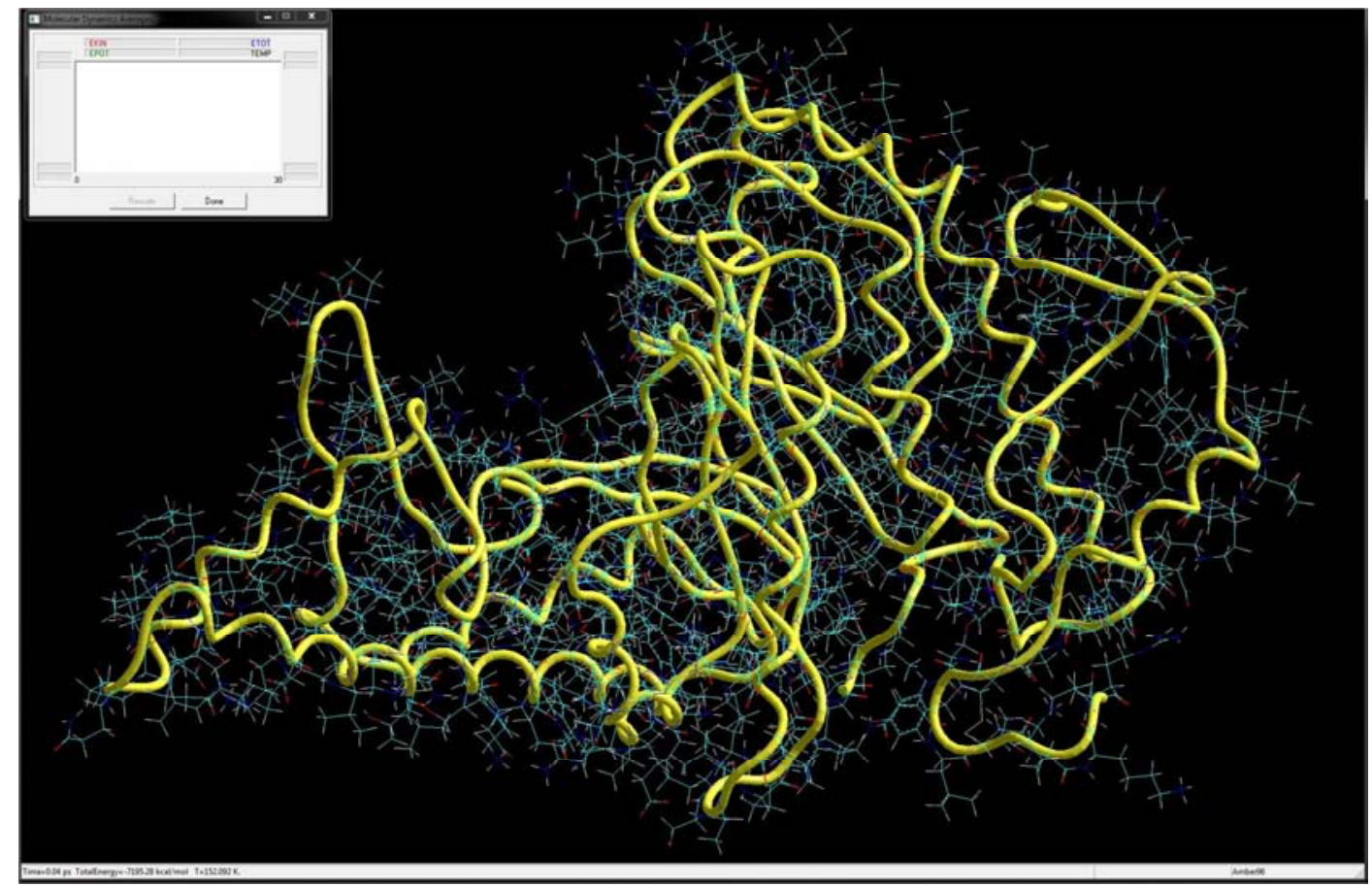

Figure 6. $3 D$ structure of free BTH (without ligands) at $340 \mathrm{~K}(0.04 \mathrm{psec})$.

The transition was the most significant between Glu-105 and Arg-59, i. e., between protein chain peak E 105 and positively charged area R 59 which represent an important initial stage in calculated denaturation of BTH. The distances between other amino acid residues which are responsible for catalytic activity of BHT and those in close proximity (Asp-147 and Asn-150, Glu-149 and Lys-162,
Glu-149 and Trp-148) did not change considerably. These data demonstrate a minor distortion of the active site; however, complete closure of the entrance for the substrate inactivated the enzyme. Docking of BTH with $\mathrm{CH}$ ligands has revealed their stabilizing effect on $3 \mathrm{D}$ structure of the enzyme at 310 (Figures 2-4), 320 and $340 \mathrm{~K}$ (in comparison with Figures 6 and 7 for free BTH) observed for 35 ns. 
Binding of the ligands at $\operatorname{ch} 6, \operatorname{ch} 3$ and ch 1 sites increased denaturation temperature by approximately $10 \mathrm{~K}$ and changed the nature of conformational transitions in BTH molecule. It should be noted that binding at ch 6 site produced a considerable stabilizing effect on the enzyme structure. Interactions between $\mathrm{BTH}$ and $\mathrm{CH}$ are based on electrostatic forces.

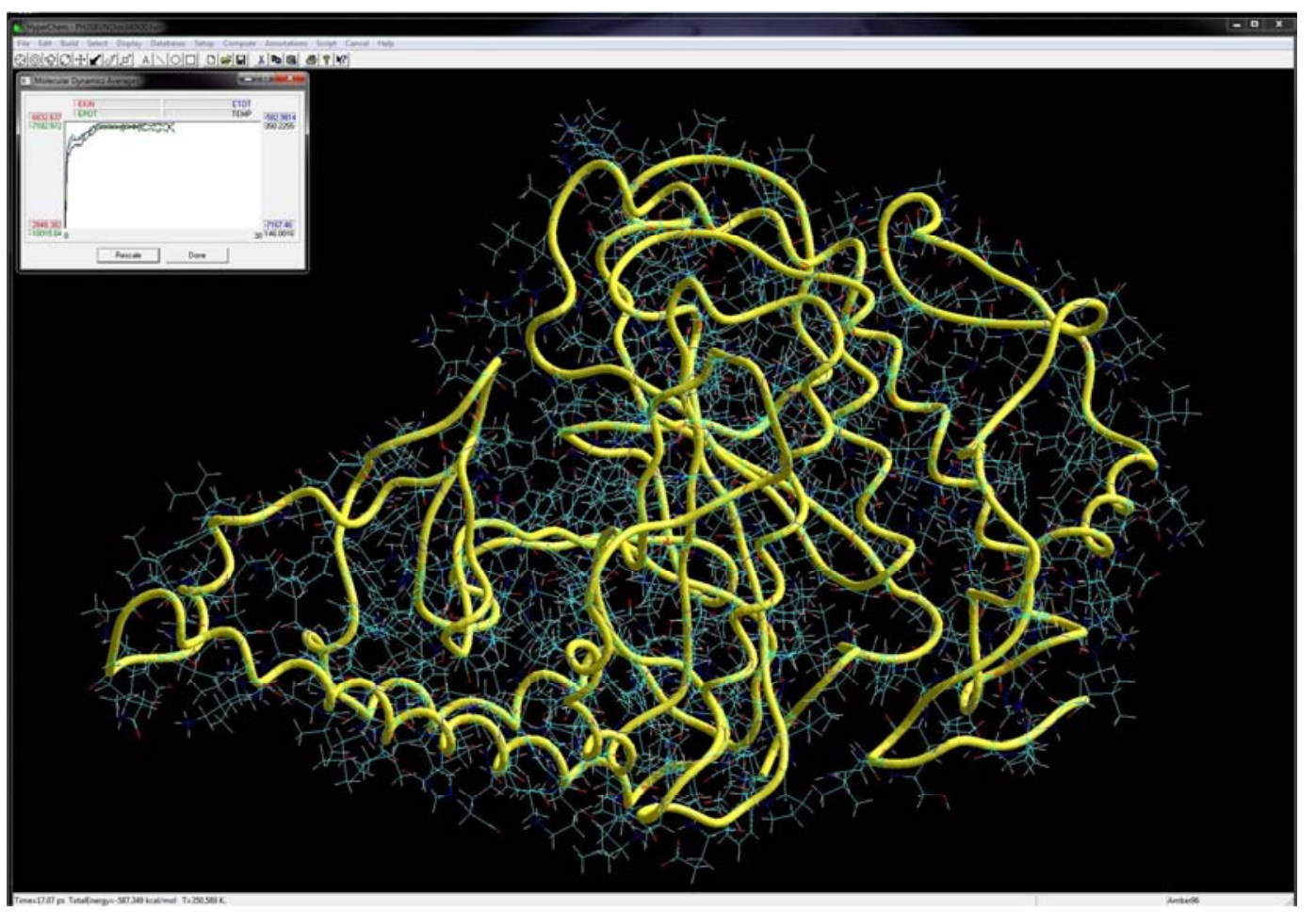

Figure 7. Narrowing of the entrance to BTH active site (which leads to the enzyme blocking), $340 \mathrm{~K}, 17 \mathrm{psec}$ calculational observation.

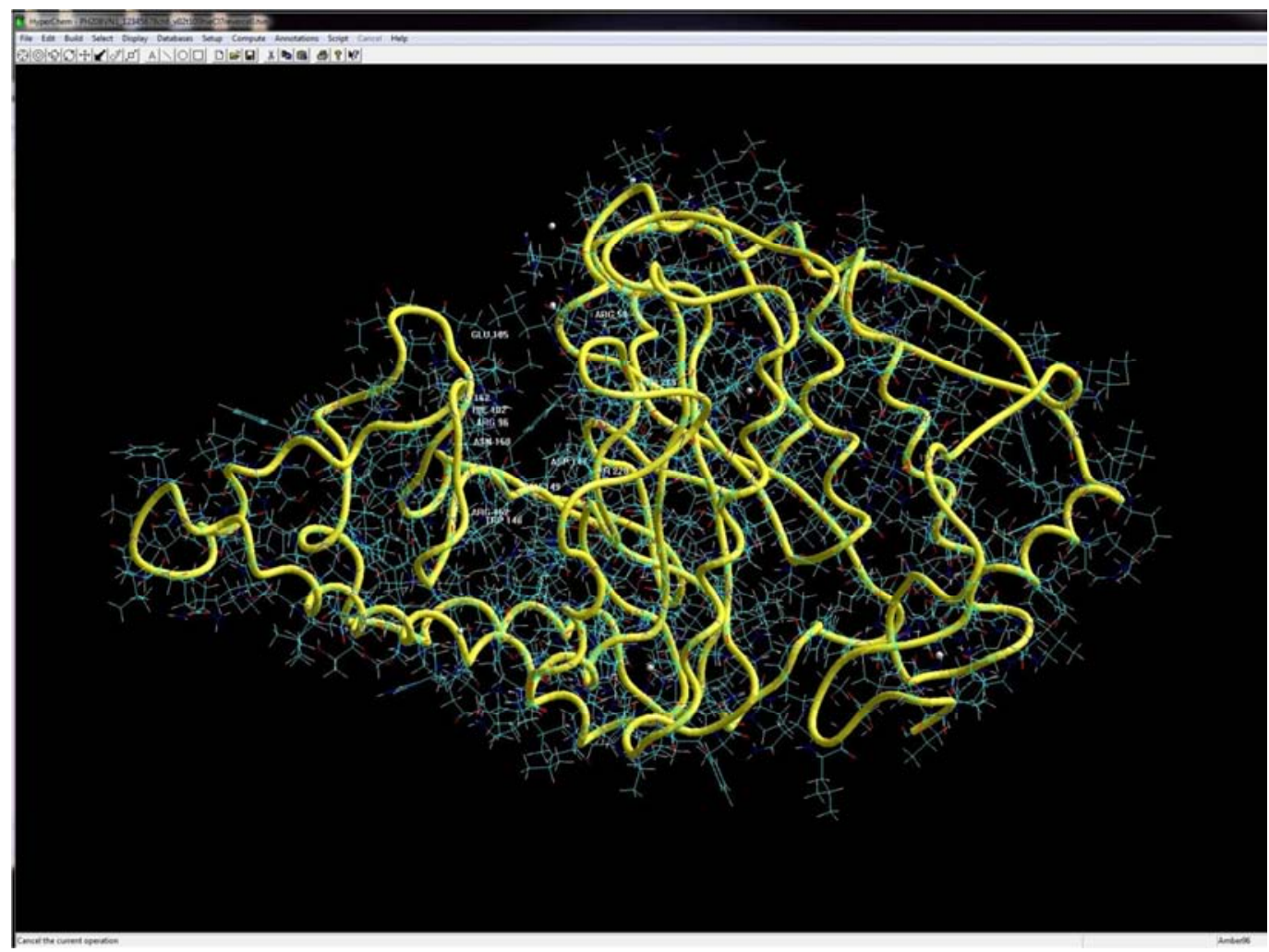

Figure 8. Reverse structural dynamics of $3 D$ model of free BTH after lowering temperature to $295 K, 25$ s. Similarity is visible between protein structures in Figures 7 and 8 and the difference from that in Figure 6 indicate irreversible deformation of BTH without CH ligands. 


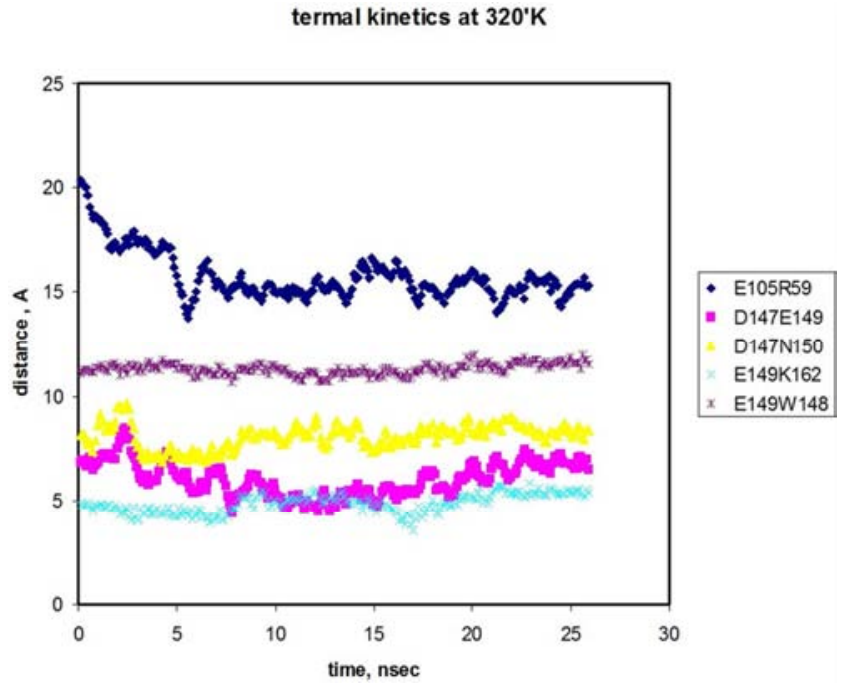

Figure 9. Changes in the distance between amino acid residues in BTH active site over calculational observation period at $320 \mathrm{~K}$.

\section{Conclusion}

Steric configuration of $\mathrm{CH}$ used in this study for docking differs from that of the specific BTH substrate hyaluronan only by configuration of $\mathrm{C}(4)$ in hexosamine residue [20]. Presumably, these delicate differences produce a considerable regulatory effect on the structure-function relationship of hyaluronidase. Our findings show that $\mathrm{CH}$ (dimer of trimer) $\mathrm{N}$-acetylated at a galactose residue but nonsulfatated stabilizes 3D structure of BTH (calculational heat inactivation) after binding predominantly at ch 6 as well as at ch 3 and ch 1 positions. The effect of $\mathrm{CH}$ is higher than that of CHS upon the enzyme inhibition by HP tetramer, which requires binding of 4-5 CHS ligands to the enzyme surface [8]. Stabilizing effects of $\mathrm{CH}$ and $\mathrm{CHS}$ ligands against heat inactivation and HP-induced inhibition, respectively, are fundamental for further direct theoretical comparative study of the effects produced by these ligands, considering the interactions between BHT and GAG in its microenvironment. Moreover, the sequancious perform of molecular docking of hyaluronidase with glycosamonoglycan ligands may contribute to founded ascertainment of modification manner of this enzyme for cardiology application.

\section{Conflict of Interest}

The authors declare that they have no conflicts of interest.

\section{Acknowledgements}

The study was financially supported by Russian Foundation for Basic Research (grant 18-015-00056) and Ministry for Health Care.

\section{List of Abbreviations}

$\begin{array}{ll}\text { 3D } & \text { space (tertiary) protein structure. } \\ \text { BTH } & \text { bovine testicular hyaluronidase. } \\ \text { GAG } & \text { glycosaminoglycan. } \\ \text { HP } & \text { heparin. } \\ \text { CH } & \text { chondroitin. } \\ \text { CHS } & \text { chondroitin sulfate. }\end{array}$

\section{References}

[1] Maksimenko AV. Development and application of targeted therapeutic protein conjugates. Russ. J. Gen. Chem. 2014; 84 (2): 357-363. Doi: 10. 1134/S1070363214020376.

[2] Maksimenko AV. Results and achievements in the engineering of pharmacological enzymes for clinical application. Med. Res. Arch. 2018; 6 (1): 1-13. http://journals.ke-i.org/index. php/mra

[3] Maksimenko AV. Translational research into vascular wall function: regulatory effects of systemic and specific factors. J. Transl. Sci. 2017; 3 (2): 1-10. Doi: 10. 15761/JTS. 1000180.

[4] Almond A. Multiscale modeling of glycosaminoglycan structure and dynamics: current methods and challenges. Curr. Opin. Struct. Biol. 2018; 50: 58-64. http://doi.org/10.1016/j.sbi.2017.11.008.

[5] Sankaranarayanan NV, Nagarajan B, Desai UR. So you think computational approaches to understanding glycosaminoglycan-protein interactions are too dry and too rigid? Think again! Curr. Opin. Struct. Biol. 2018; 50: 91-100. http//doi.org/10.1016/j.sbi.2017.12.004

[6] Hage KE, Brickel S, Hermalin S, Gaulier G, Schmidt C, Bonacina L, van Keulen SG, Bhattacharyya S, Chergui M, Hamm P, Rothlisberger U, Wolf J-P, Meuwly M. Implication of short time scale dynamics on long time process. Struct. Dyn. 2017; 4: 061507. http://doi.org/10.1063/1/499648

[7] Meng X-Y, Zhang H-X, Mezei M, Cui M. Molecular docking: a powerful approach for structure-based drug discovery. Curr. Comput. Aided Drug Des. 2011; 7 (2): 146-157.

[8] Reitsma S, Slaaf DW, Vink H, van Zandvoort MAMJ, onde Egbrink MGA. The endotheliumglycocalyx: composition, function, and visualization. Pflügers Arch. 2007; 454: 345-359.

[9] Broekhuisen LN, Moojij HL, Kastelein JJ, Stroes ESG, Vink H, Nieuwdorp M. Endothelial glycocalyx as potential diagnostic and therapeutic target in cardiovascular disease. Curr. Opin. Lipidol. 2009; 20: 57-62.

[10] Maksimenko AV, Schechilina YV, Tischenko EG. Resistance of dextran-modified hyaluronidase to inhibition by heparin. Biochemistry (Moscow). 2001; 66 (4): 456-463.

[11] Maksimenko AV, Schechilina YV, Tischenko EG. Role of the glycosaminoglycan microenvironment of hyaluronidase in regulation of its endoglycosidase activity. Biochemistry (Moscow). 2003; 68 (8): 862-868.

[12] Meyer K, Rapport MM. The hydrolysis of chondroitin sulfate by testicular hyaluronidase. Arch. Biochem. 1950; 27 (2): 287-293. 
[13] Hoffman P, Meyer K, Linker A. Transglycosylation during the mixed digestion of hyaluronic acid and chondroitin sulfate by testicular hyaluronidase. J. Biol. Chem. 1956; 219 (2): 653-663.

[14] Maksimenko AV, Beabealashvili RS. Conformational transitions in $3 \mathrm{D}$ model of bovine testicles hyaluronidase during molecular docking with glycosaminoglycan ligands. Russ. J. Bioorgan. Chem. 2018; 44 (2): 165-172.

[15] Maksimenko AV Turashev AD, Beabealashvili RS. Stratification of chondroitin sulfate binding sites in 3D-model of bovine testicular hyaluronidase and effective size of glycosaminoglycan coat of the modified protein. Biochemistry (Moscow). 2015; 80 (3): 284-295.

[16] Pettersen EF, Goddard TD, Huang CC, Couch GS, Greenblatt DM, Meng EC, Ferrin TE. UCSF Chimera - a visualization system for exploratory research and analysis. J. Comput. Chem. 2004; 25 (13): 1605-1612.
[17] Sanner MF, Olson AJ, Spehner JC. Reduced surface: an efficient way to compute molecular surfaces. Biopolymers. 1996; 38 (3): 305-320.

[18] Lang PT, Brozell SR, Mukherjee S, Pettersen EF, Meng EC, Thomas V, Rizzo RC, CaseDA, James TL, Kuntz ID. DOCK 6: combining techniques to model RNA-small molecule complexes. RNA. 2009; 15 (6): 1219-1230.

[19] Yang J, Chi L. Characterization of structural motifs for interactions between lycosaminoglycans and proteins. Carbohyd. Res. 2017; 452: 54-63.

[20] Maksimenko AV, Beabealashvili RS. Effect of the hyaluronidase microenvironment on the enzyme structure-function relationship and computational study in silico of the molecular docking of chondroitin sulfate and heparin short fragments to hyaluronidase. Russ. Chem. Bull. Intl. Ed. 2018; 67 (4): 1-11. 